\title{
Current Disease-Modifying Therapies in Multiple Sclerosis
}

\author{
Bernd C. Kieseier, M.D., ${ }^{1}$ and Hans-Peter Hartung, M.D. ${ }^{1}$
}

The publisher regrets an error in Table 2 of the above article in Seminars in Neurology, Volume 23, Number 2, p. 142. On the last line of column one, under the heading "Clinical Subform," the entry Primary-relapsing $M S$ should read Relapsing progressive MS. The corrected table appears below.

Table 2 Current Treatment Strategies in Multiple Sclerosis (MS)

\begin{tabular}{ll}
\hline Clinical Subform & Therapy \\
\hline Acute relapse & $\begin{array}{l}\text { Pulsed high-dose glucocorticosteroids } \\
\text { Escalation: Plasma exchange } \\
\text { Interferon beta }\end{array}$ \\
$\begin{array}{l}\text { Clinically isolated syndromes (CIS) with high } \\
\text { risk of developing clinically definite MS }\end{array}$ & First line: Interferon beta, glatiramer acetate \\
Relapsing-remitting MS & Second line: IVIG, azathioprine \\
& With severe relapses and progression: Mitoxantrone \\
Secondary progressive MS & With relapses: Interferon beta \\
& Progressive: Mitoxantrone \\
Primary progressive MS & Second line: Cyclophosphamide \\
Relapsing progressive MS & No established therapy available \\
\hline
\end{tabular}

Neuroimmunotherapy; Editor in Chief, Karen L. Roos, M.D.; Guest Editor, Marinos C. Dalakas, M.D. Seminars in Neurology, Volume 23, Number 2, 2003. Address for correspondence and reprint requests: Bernd C. Kieseier, M.D., Department of Neurology, Heinrich-HeineUniversity, Moorenstrasse 5, 40225 Düsseldorf, Germany. ${ }^{1}$ Department of Neurology, Heinrich-Heine-University, Düsseldorf, Germany. Copyright (C) 2003 by Thieme Medical Publishers, Inc., 333 Seventh Avenue, New York, NY 10001, USA. Tel: +1(212) 584-4662. 0271$8235, \mathrm{p} ; 2003,23,03,343,344, \mathrm{ftx}, \mathrm{en} ; \sin 00265 \mathrm{x}$. 
